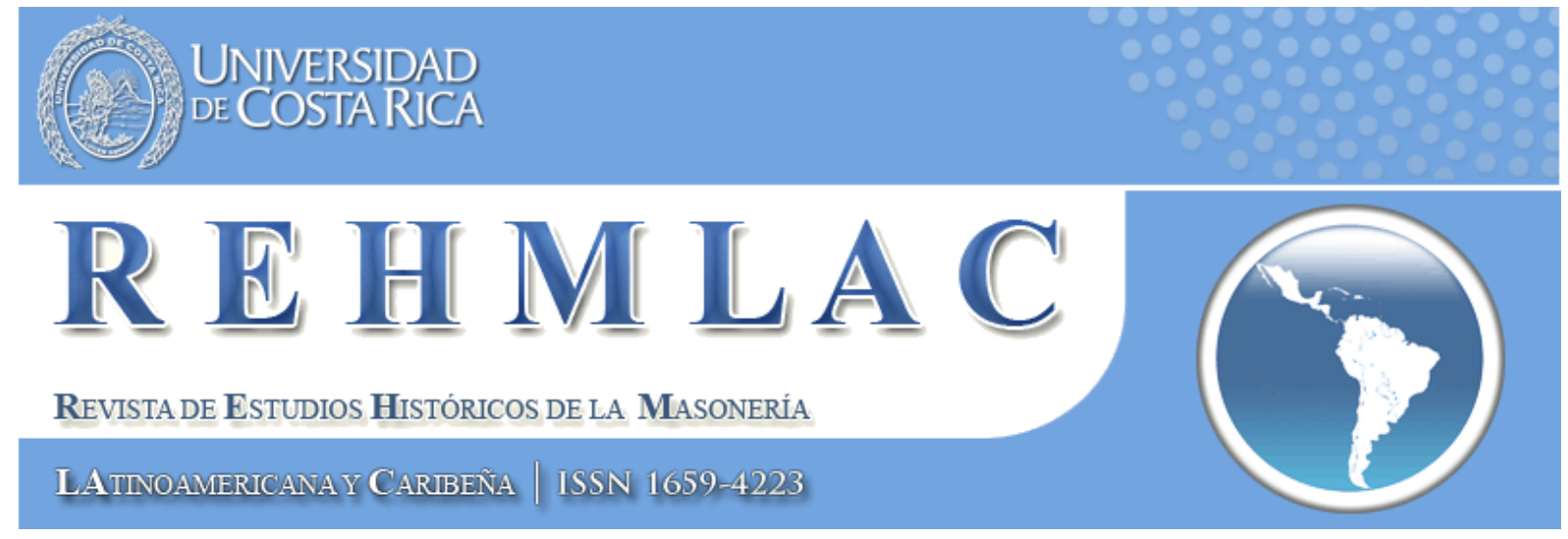

« Le système-monde maçonnique à la veille de la Première Guerre mondiale: une analyse archéologique »

\author{
Dévrig Mollès
}

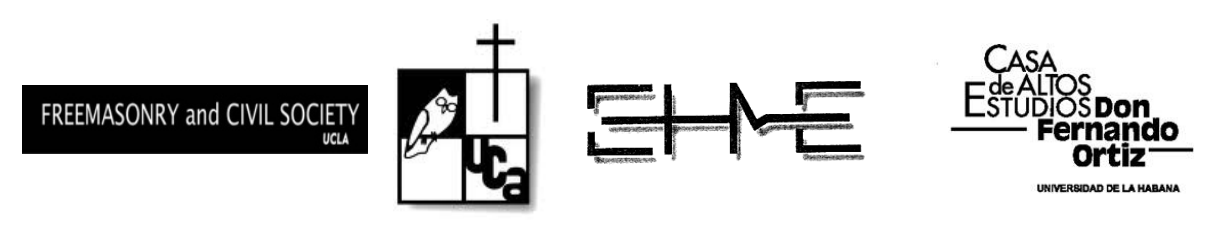


(C) Dévrig Mollès et REHMLAC.

\section{Équipe REHMLAC}

Conseil scientifique :

Miguel Guzmán-Stein (Universidad de Costa Rica, Costa Rica)

José Antonio Ferrer Benimeli (Universidad de Zaragoza, España)

Margaret Jacob (University of California Los Angeles, United States)

Eduardo Torres Cuevas (Universidad de La Habana, Cuba)

María Eugenia Vázquez Semadeni (Stanford University, United States)

Eric Saunier (Université du Havre, France)

Andreas Önnerfors (Lunds universitet, Sverige)

Samuel Sánchez Gálvez (Universidad Carlos Rafael Rodríguez de Cienfuegos, Cuba)

Roberto Valdés Valle (Universidad Centroamericana “José Simeón Cañas”, El Salvador)

Céline Sala (Université de Perpignan, France)

Dominique Soucy (Université de Franche-Comté, France)

Guillermo de los Reyes Heredia (University of Houston, United States)

Dévrig Mollès (Université de Strasbourg, France)

Felipe Santiago del Solar Guajardo (Universidad ARCIS, Santiago de Chile)

Carlos Francisco Martínez Moreno (Universidad Nacional Autónoma de México, México)

Michel Goulart da Silva (Universidade do Estado de Santa Catarina, Brasil)

Examinateurs de style:

Sylvia Hottinger (Centro de Estudios Históricos de la Masonería Española, España)

Kyle Jackson (University of California Los Angeles, United States)

Web administrateur et designer:

Luis Martín Valverde Alfaro (Universidad de Costa Rica, Costa Rica)

Editor:

Yván Pozuelo Andrés (IES Universidad Laboral de Gijón, España)

E-mail: yvan@edu.xunta.es

Director:

Ricardo Martínez Esquivel (Universidad de Costa Rica, Costa Rica)

E-mail: ricardo.martinezesquivel@ucr.ac.cr

Adresse web : www.rehmlac.com/

E-mail : info@rehmlac.com

PO Box : 243-2300 San José, Costa Rica 
Cité dans :

Academia.edu

Aladin. WRLC. Libraries Catalog

AFEHC. Asociación para el Fomento de los Estudios Históricos en Centroamerica

Biblioteca de Georgetown

CRICCAL, Université Sorbonne Nouvelle Paris 3

CERGE EI. Portál elektronických časopisů. Univerzita Karlova v Praze

Departamento de Filosofía de la Universidad Centroamericana "José Simeón Cañas"

Dialnet, Universidad de la Rioja

Directorio y recolector de recursos digitales del Ministerio de Cultura de España

DOAJ. Directory of Open Access Journals

e-revist@s. Plataforma Open Access de Revistas Científicas Electrónicas

Freemasonry and Civil Society Program at UCLA

Fudan University Library Academic Resource Portal

Google académico

Institute for the Study of the Americas at University of London

Latindex (UNAM)

Latindex.ucr. Repositorio de revistas de la Universidad de Costa Rica

Library Catalogue of University of South Australia

Museo Virtual de la Historia de la Masonería de La UNED

Nuevo Mundo. Mundos Nuevos

REDIAL. Red Europea de Información y Documentación sobre America Latina

SciELO - Scientific Electronic Library Online

Sherpa/Romeo

SID. Sistema Integrado de Documentación. Universidad Nacional de Cuyo

Toronto Public Library

UBO. Revues en ligne. Université de Bretagne Occidentale

Universia. Biblioteca de Recursos

University of Saskatchewan Library

University of Wiscosin-Madison Libraries

Western Theological Seminary. Beardslee Library Journals 


\title{
« Le système-monde maçonnique à la veille de la Première Guerre mondiale: une analyse archéologique »
}

\author{
Dévrig Mollès \\ Docteur en Histoire, Université de Strasbourg, France. Director de Archivo de la Gran Logia Argentina. \\ E-mail : devrigmolles@gmail.com
}

Date de réception: 3 août 2014 - Date d'acceptation: 23 novembre 2014

Mots-clé
Première Guerre mondiale, franc-maçonnerie, système-monde, statistiques, carte, graphiques

Keywords

World War I, freemasonry, word-system, statistics, map, graphs

\section{Résumé}

Cet article trouve son origine dans une question : de quoi parle-t-on exactement lorsqu'on évoque la franc-maçonnerie internationale à la veille de la Première Guerre mondiale? Quels étaient le volume, les superficies, les dimensions du système-monde maçonnique en 1914? Quels étaient ses équilibres géopolitiques et géoculturels ? Ce texte présente donc une photographie prise à un moment-clé.

\begin{abstract}
This article finds its origin in a question: what are we talking about exactly when we evoke the concept of international freemasonry on the eve of the World War I? What were the volume, surfaces, and dimensions of the masonic world-system in 1914? What were its geopolitical and geocultural balances? This text thus presents a snapshot taken at that key moment.
\end{abstract}

\section{Introduction}

Le $\mathrm{XIX}^{\mathrm{e}}$ siècle fut l'âge d'Or de la civilisation libérale. Les réseaux maçonniques en furent l'un des fleurons: apparus au XVIIIe siècle, ils furent l'un prototype fondateur de l'opinion publique internationale. Dans le sillage de l'expansion des puissances et des sociétés occidentales, ils se ramifièrent et constituèrent un système-monde ${ }^{1}$. La Première Guerre mondiale marqua, on le sait, un basculement, précipitant la civilisation libérale et toutes les institutions qui en étaient issues dans une profonde crise. Avant d'aborder, dans de futurs travaux, cette crise globale au regard de la question maçonnique, cette période charnière semble devoir être analysée en profondeur.

Dans un premier temps, une question semble devoir être résolue : de quoi parle-t-on exactement lorsqu'on évoque la franc-maçonnerie internationale à la veille de la Première Guerre mondiale? Quels étaient son volume, sa superficie, ses dimensions ? Quels étaient ses équilibres géopolitiques et géoculturels? Quelle y était enfin la position de l'Amérique latine?

\footnotetext{
${ }^{1}$ Pour une analyse de la formation de ce système-monde maçonnique, voir Dévrig Mollès, «Le Triangle Atlantique: Émergence et Expansion de La Sphère Maçonnique Internationale. Une Analyse Statistique (1717-1914) », dans: Nuevo Mundo Mundos Nuevos. Nouveaux Mondes Mondes Nouveaux - Novo Mundo Mundos Novos - New World New Worlds 14 (2014 [cité 17 Décembre, 2014]): disponible dans http://nuevomundo.revues.org/67498
} 
Carte 1

La morphologie territoriale du système-monde maçonnique a la veille de la Première Guerre mondiale

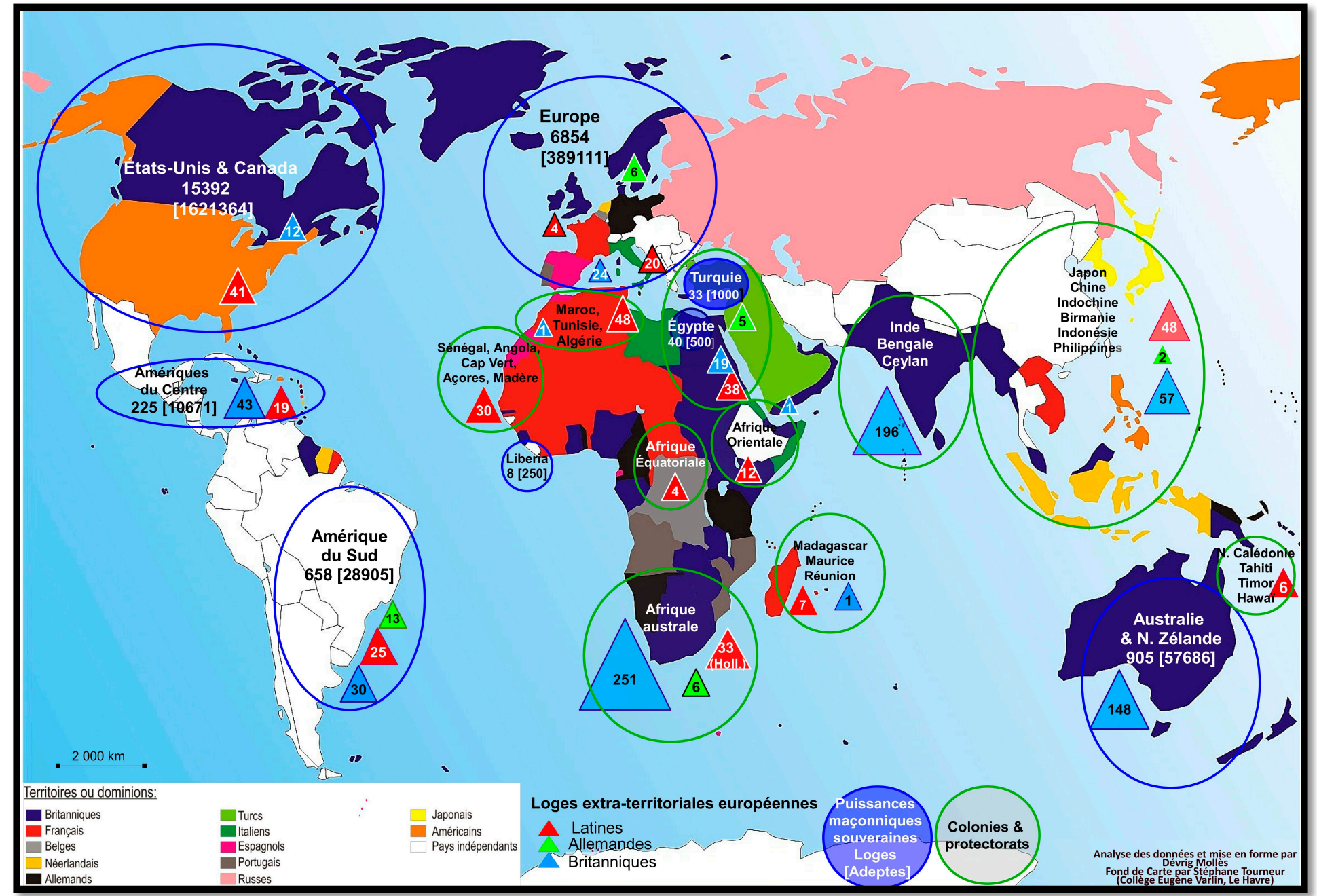

Source : Bureau International de Relations Maçonniques, Annuaire de la Maçonnerie Universelle 8 (Berne, Suisse: Imp. Büchel, 1914), 460 pages. 


\section{Sources et méthodes}

Ces questions semblent échapper à l'historiographie universitaire, généraliste ${ }^{2}$ ou spécialisée $^{3}$, et aussi aux historiographies militantes. Il faut donc "inventer" des sources susceptibles d'alimenter une sorte d'archéologie historique: la reconstruction, à partir de ces vestiges documentaires, d'un vaste système de pôles et de réseaux. La huitième et dernière édition de l'Annuaire de la Maçonnerie Universelle constitue une pièce fondamentale, méconnue et -à ma connaissance- jamais analysée. Publié en 1914 par le Bureau International de Relations Maçonniques, pourvu de presque 500 pages, cet Annuaire permet-il de reconstruire l'iceberg maçonnique international ? Le Bureau International devait en effet:

... faciliter les relations fraternelles entre les Puissances maçonniques [...] Recueillir tous les renseignements sur l'organisation et l'activité de la Maçonnerie universelle [...] Établir la liste des Grands Orients, Grandes Loges et Sup. Cons. [...] Cataloguer les journaux maçonniques périodiques de tous les pays [...] Réunir les documents nécessaires pour l'histoire abrégée de la Maçonnerie universelle $[\ldots]^{4}$

On peut donc se réjouir de l'existence de cette source. Mais quelle est sa fiabilité ? Peut-on lui accorder un crédit illimité ? Comme tout document écrit ou oral, ces Annuaires ont été fabriqués par des acteurs dotés d'informations partielles et d'intentions partiales. Quelles étaient celles du Bureau International ? L'esprit critique s'impose.

Une première limite est à noter d'emblée : l'eurocentrisme. Ce trait de mentalité imprègne les documents utilisés ici. La liste de "dates importantes pour l'Histoire de la Maçonnerie ${ }^{5}$ en constitue un bel exemple. Cette chronologie concernait essentiellement les Îles britanniques, la France, l'Allemagne et, dans une moindre mesure, les États-Unis. Les références à l'Amérique du Sud y étaient très rares. Le reste du monde n'existait pas. La liste des francs-maçons célèbres reproduisait le même schéma: elle mentionnait deux cents vingt-six Européens (la plupart Britanniques, Français, Belges et Européens du Nord), cinq États-uniens et un Brésilien ${ }^{6}$. Doit-on en conclure que 1'Europe occidentale, l'Allemagne, les

\footnotetext{
${ }^{2}$ L'historiographie universitaire généraliste a récemment été analysée -à travers de grands ouvrages d'histoire comparée- par Dévrig Mollès, "L'histoire Globale et La Question Maçonnique: Éléments Pour Une Analyse », dans: REHMLAC 6, no. 1 (May-Novembre 2014): 3-33 [cité 3 Octobre, 2014]: disponible dans http://rehmlac.com/recursos/vols/v6/n1/rehmlac.vol6.n1-dmolles.pdf

${ }^{3}$ L'historiographie spécialisée a récemment été analysée, pour l'Amérique latine, par José Antonio Ferrer Benimeli, "Aproximación a La Historiografía de La Masonería Latinoamericana," dans: REHMLAC 4, no. 1 (May-Novembre 2012): 2-120 [cité 3 Octobre, 2013]: disponible dans http://rehmlac.com/recursos/vols/v4/n1/rehmlac.vol4.n1-jferrer.pdf. Ricardo Martínez Esquivel, "Hacia la construcción de una historia social de la masonería en Centroamérica," dans: Estudios 27 (2013 [cité 3 Octobre, 2013]): disponible dans http://revistas.ucr.ac.cr/index.php/estudios/article/view/12703/11951

4 «Programme» du Bureau International de Relations Maçonniques, dans: Annuaire de la Maçonnerie Universelle 8 (Berne, Suisse: Imp. Büchel, 1914), 38-40. Ce document sera désigné sous l'abréviation de BIRM, 1914. La graphie et les majuscules sont conformes, comme toujours, à celles de l'original.

5 BIRM, 1914, 41-46. Les majuscules appartiennent, comme toujours, au document original.

${ }^{6}$ BIRM, 1914, 46-51: Au total étaient cités deux cents vingt-six Européens (la plupart Britanniques, Français, Belges et Européens du Nord), cinq États-Uniens [Benjamin Franklin (1706-1790), George Washington
} 
États-Unis avaient été entre 1700 et 1914 les seuls pôles de production maçonnique ? Presque absente de ce palmarès, l'Amérique latine constituait pourtant, depuis le début du XIX ${ }^{\mathrm{e}}$ siècle, le troisième pôle de cette sphère internationale ${ }^{7}$.

Seconde limite : le "régularisme". Le Bureau International de Relations Maçonniques refusait en principe de se prononcer «sur la régularité ou l'irrégularité [...] des Grands Orients, Grandes Loges et Sup. Cons. »; il se refusait à « classer les Maçonneries diverses par rang de valeur ${ }^{8}$ ? En cela, il répondait à l'esprit libéral et œcuménique de ses fondateurs. En 1889, à l'occasion du premier Centenaire de la Révolution française, des Français, des Belges et des Suisses avaient appelé de leurs vœux la création d'un «tribunal international des communications fraternelles $»^{9}$, selon le mot lancé par Frédéric Desmons -pasteur protestant, sénateur radical et membre influant du Grand Orient de France. Fondé en 1902, le Bureau International possédait deux objectifs centraux: rapprocher Européens et Américains, mais aussi Latins et Anglo-Saxons. La gestion en fut confiée aux Suisses de la Grande Loge Alpina qui, de leur propre aveu, échouèrent ${ }^{10}$. La tentative les amena à publier des Annuaires d'une grande richesse, mais aussi à d'amères concessions. En 1903, par exemple, ils avaient amorcé une liaison afro-américaine, suscitant la protestation indignée des francs-maçons blancs, anglo-saxons et protestants des États-Unis. Le Bureau recula, s'excusant de son « inexpérience » face aux « clandestins » ${ }^{11}$ qui furent expulsés des Annuaires. Comme les femmes, les noirs étaient indésirables aux yeux de ceux qu'on voulait séduire. Ainsi, seules «les Puissances maçonniques régulières ${ }^{12}$ furent recensées dans l'Annuaire du Bureau International de Relations Maçonniques. Qu'est-ce que cela signifiait ? Le Bureau possédait un objectif diplomatique plutôt qu'une vocation scientifique; il tentait de concilier les deux courants géoculturels dominants de l'espace maçonnique international. Souhaitant amadouer les obédiences anglo-saxonnes les plus "régularistes", 1'Annuaire de la Maçonnerie Universelle ne parlait que d'une partie -certes largement majoritaire- des loges masculines. Il évacuait une myriade d'hétérodoxies masculines ${ }^{13}$, afro-américaines ${ }^{14}$, féminines ou mixtes

(1732-1799), Charles W. Moore (1801-1873), Albert Pike (1808-1883), Abraham Lincoln (1809-1865)] et un Brésilien [Pedro Ier d'Alcantara, empereur du Brésil (1798-1834)].

7 Voir Mollès, « Une Analyse Statistique (1717-1914)».

${ }^{8}$ Bernard Perrelet, Le Bureau International de Relations Maçonniques: Organisation, but, activité (Berne Neuchâtel: Bureau International des Relations Maçonniques - Imprimerie Büchler \& Co., 1913), 8.

${ }^{9}$ Discours de Frédéric Desmons lors de la création du BIRM en 1902 (Genève), dans : Perrelet, Le Bureau International de Relations Maçonniques, 5-6.

${ }^{10}$ Voir Dévrig Mollès, "Triangle atlantique et triangle latin: 1'Amérique latine et le système-monde maçonnique (1717-1921) (Éléments pour une histoire des opinions publiques internationales)" (Thèse de doctorat en histoire contemporaine, Université de Strasbourg, 2012), chap. 2, 5 (notamment pp. 242-52, 553-9).

${ }^{11}$ Le Bureau international de relations maç.:. Son histoire (1889-1905), Rapport présenté à la Grande Loge Suisse Alpina le 25 juin 1905 (Berne, Suisse: Bureau International des Relations Maçonniques - Imprimerie Büchler \& Co., 1905), $31-5$.

12 BIRM, 1914, 38 et 62-366.

13 On pense, pour l'Amérique latine à cette date, à de nombreux courants alternatifs particulièrement importants, tels que le Rito Azul ou le Rito Nacional Argentino en Argentine, le Rito Nacional Mexicano au Mexique.

${ }_{14}$ Sur les franc-maçonneries afro-américaines: Cécile Révauger, Noirs et francs-maçons aux États-Unis (Paris: Dervy, 2014). Mollès, « Une Analyse Statistique (1717-1914) », 20-21 (L’Amérique anglo-saxonne). 
(qui n'étaient alors qu'à leur premiers pas) ${ }^{15}$. Ce recensement devra donc être complété dans le futur. Il faudra pour cela inventer de nouvelles sources. En l'état, cependant, j'estime qu'il couvrait au moins $95 \%$ de l'iceberg maçonnique international.

Notons toutefois que, pour le Bureau, ce régularise était un opportunisme et non un credo. Les pages de son Annuaire réunissaient d'ailleurs des institutions qui ne se parlaient pas, telles que les trois Grandes Loges britanniques et les deux fédérations recensées pour la France (le Grand Orient et la Grande Loge). Aussi quelques "groupements divers non régularisés » ${ }^{16}$ furent l'objet d'une section marginale. D'autres enfin étaient mentionnés presque par hasard, parce qu'ils maintenaient des relations bilatérales avec certaines organisations recensées. Parmi les seize groupements masculins «non régularisés ${ }^{17}$ : huit résidaient au Mexique ${ }^{18}$; deux se trouvaient en Italie ; enfin, un groupement "irrégulier" était recensé respectivement pour l'Argentine (qui en comptait réellement au moins cinq ${ }^{19}$ ), Cuba, le Salvador, la Colombie, la Turquie, l'Espagne et le Luxembourg. Il faut donc conserver une distance critique par rapport à ces données, afin peut-être d'en dépasser les limitations.

Troisième limite: la typologie des loges, dont la multiplicité pose problème ${ }^{20}$. L'historien et le sociologue -conditionnés par leurs matériaux documentaires- travaillent généralement sur des loges "institutionnelles", dont l'activité est régulée par des règles communes à une fédération déterminée (Grands Orients ou Grandes Loges notamment). Volontairement soumises à la régulation d'une "obédience" qui appliquait des règles théoriquement uniformes, ces loges produisaient une documentation théoriquement conservée par une bureaucratie centrale. Ces loges et obédiences étaient donc des cristallisations institutionnelles flottant à la superficie de réseaux fluctuants. En revanche, d'autres types de loges échappent au radar; on ne devine leur présence qu'au travers de reflets indirects. Leur activité n'est généralement pas ou peu documentée. Les loges "couvertes" -créées dans un but déterminé, généralement politique ou économique, au sein d'une obédience- ne figuraient pas sur les registres du Bureau International de Relations Maçonnique, même si elles jouèrent parfois un rôle important. D'autres types de loges semblaient échapper à l'Annuaire de la Maçonnique Universelle. C'est le cas, par exemple, des loges militaires et navales, nombreuses dans les régiments britanniques par exemple ${ }^{21}$ et dont le modèle fut semble-t-il

15 On pense aux organisations maçonniques féminines et mixtes (notamment l'Ordre International Mixte Le Droit Humain, fondé en France à la fin du XIX ${ }^{\mathrm{e}}$ siècle et diffusé dans le monde anglo-saxon en marge des courants maçonniques majoritaires en Angleterre, en Inde et en Amérique): voir Cécile Révauger, « Les Femmes et La Franc-Maçonnerie, Des Origines À Nos Jours », REHMLAC 4, no. 2 (Décembre 2012-Avril 2013): 11-31 [cité 3 Octobre, 2013]: disponible dans http://rehmlac.com/recursos/vols/v4/n2/rehmlac.vol4.n2-crevauger.pdf

16 BIRM, 1914, 367-374.

17 BIRM, 1914, 367-374.

18 Pour le Mexique étaient citées, sans aucunes précisions statistiques ni chronologiques, les grandes loges de Veracruz (Unida Mexicana), de Puebla, de Oaxaca, de Tamaulipas, de Nuevo León, de Coahuila, de Chihuahua et Valle de México.

19 Mollès, "Triangle atlantique," chap. 4, 5.

${ }^{20}$ Un article sur la typologie des "Loges" dans. Daniel Ligou, ed., Dictionnaire de la franc-maçonnerie (Paris : Presses Universitaires de France, 1998), 729-736.

${ }^{21}$ Comme point de départ, on peut consulter l'ouvrage récent de Cécile Révauger \& Éric Saunier, analysé par Ferrer Benimeli, "Reseña de La Franc-Maçonnerie dans les ports, editado por Cécile Révauger y Éric Saunier", REHMLAC 5, no. 1 (May-Novembre 2013): 228-236 [cité 3 Octobre, 2014]: disponible dans 
transféré en Amérique latine sous la forme de loges ferroviaires, apparues à la fin du XIXe siècle $^{22}$ dans le sillage de la multiplication des voies ferrées. Mentionnons finalement les loges d'exil: souvent autonomes, elles ne s'intégrèrent pas nécessairement à une fédération et se couvrirent éventuellement du voile de la clandestinité. Toutes les loges -fédérées ou autonomes- contribuèrent à l'expansion de la sphère maçonnique dans des régions souvent improbables. Toutefois, ces loges "autonomes" revêtent un intérêt plus ponctuel et plus local, lorsqu'il s'agit d'étudier une situation déterminée. Elles ne sauraient faire basculer les statistiques présentées ici.

Enfin, l'Annuaire du Bureau International de Relations Maçonniques s'alimentait des informations que voulaient bien lui fournir la centaine de fédérations nationales qui y étaient répertoriée. Cette variable importante conduit à ne pas y chercher une absolue exactitude.

En synthèse, le regard du Bureau International était eurocentriste et régulariste. Centré sur l'Europe et les États-Unis, et dans une moindre mesure sur l'Amérique Latine et l'Australie, il excluait les franc-maçonneries alternatives. Néanmoins, dans le registre statistique et archéologique, l'Annuaire de la Maçonnerie Universelle constitue la source la plus complète et la plus panoramique disponible. Ses données sont raisonnablement fiables. Il est le point de départ indispensable, relativement exhaustif, même si ses lacunes appellent de futurs compléments. il autorise une analyse panoramique.

Quel était le volume, les couleurs et les équilibres de la sphère maçonnique internationale à la veille de la Première Guerre mondiale? Pour répondre à cette question, il a été nécessaire de construire patiemment une base de données informatique. Actuellement, cette base est composée de quatre rubriques dédiées respectivement aux "fédérations nationales", aux "loges extraterritoriales", aux "loges nationales-migratoires" et enfin aux "rites et systèmes particuliers" ${ }^{23}$. Elle a été construite autour de champs relatifs aux territoires, aux langues, aux filiations géoculturelles. Elle comprend enfin des données précises, chiffrées et datées et des informations diverses. Ces données ont autorisé la réalisation de cartes et de

http://rehmlac.com/recursos/vols/v5/n1/rehmlac.vol5.n1-jferrer.pdf. Signalons sur ce sujet l'important ouvrage de Robert Freke Gould, Military Lodges: The Apron and the Sword or Freemasons under Arms. Accounts of Lodges in Regiments and Ships of War and Famous Soldiers and Sailors of All Countries Who Have Belonged to the Society, Together with Biographies of Distinguished Military and Naval Brethren, and Anecdotes Showing the Influence of Masonry in Warfare (London: Gale \& Polden Ltd., 1899).

${ }^{22}$ Repérables par exemple en Argentine entre la fin du XIXe siècle et la première moitié du XXe siècle : «Je me rappelle avec émotion [...] l'épisode de mon initiation, le plus grand de ma vie profane et maçonnique. Je ne me souviens pas exactement combien de temps s'est écoulé depuis [...] Elle se produisit dans une loge de campagne, formée par des ouvriers, la plupart machinistes et cheminots [...] Cette nuit fut pour moi la révélation d'un monde nouveau. J'avais durement lutté durant les premières années de ma vie [...] pour la première fois de ma vie, je vis sur ces visages d'ouvriers un regard chaleureux d'affection [...] Pour la première fois je sentis cet amour pour l'humanité [...]» (Témoignage de Jorge Coen, "Recuerdos de Mi Iniciación”, dans : Verbum, Revista Masónica Argentina Fundada En 1934 1, no. 6 (Novembre 15, 1934): 4.

${ }^{23}$ Les "fédérations nationales" étaient des organisations maçonniques souveraines et indépendantes, installées en premier lieu sur le territoire politique d'un État-nation déterminé. Les "loges extraterritoriales" dépendaient d'une fédération nationale déterminée mais résidaient en territoire étranger (État-nation, protectorat ou colonie). Les "loges nationales-migratoires" étaient fédérées à une obédience nationale mais émanait d'une communauté étrangère établie dans le pays concerné (utilisant parfois la langue de cette communauté étrangère dans leurs travaux). Les "rites et systèmes particuliers" étaient des systèmes liturgiques régulant d'autres grades que les trois premiers grades communs à tous les systèmes maçonniques. 
graphiques. Ici, le niveau d'analyse restera volontairement global, réservant les perspectives régionales et nationales à de prochaines publications.

\section{Les équilibres géopolitiques}

Quel était le volume global du monde maçonnique en 1914 ? Selon l'Annuaire du Bureau International de Relations Maçonniques, «en 1913, la Maçonnerie universelle compt[ait] 23.812 loges et 2.095.627 membres $\gg{ }^{24}$.

Cet ensemble puisait ses racines dans 40 pays et s'organisait en 105 grandes loges ou grands orients. Il comptabilisait uniquement les loges dites «symboliques » qui -pratiquant les trois premiers grades d'apprenti, compagnon et maître- constituaient la base commune à tous les systèmes et rites particuliers. Certes, on l'a vu, ces chiffres étaient sous-estimés, en raison des limitations et exclusions antérieurement évoquées. Mais le poids démographique des franc-maçonneries mixtes, féminines et afro-américaines, notamment, pouvait-il remettre en question l'économie générale du monde maçonnique? Au contraire, on peut penser que l'Annuaire couvrait l'essentiel -au moins 90\%- de cet iceberg jusque-là insondé. Inversement, on peut observer que le traitement informatique des données -long et fastidieux mais nécessaire- met en évidence quelques erreurs de calculs ${ }^{25}$. Une estimation corrigée aboutit aux chiffres de 24115 loges et 2108827 membres: une différence somme toute minime au regard de l'échelle envisagée (303 loges et 13200 membres). Comment se structurait cet ensemble ? Quelles étaient les fabriques de la production maçonnique mondiale?

24 BIRM, 1914, 393.

25 Par exemple, pour le cas des États-Unis, l'Annuaire annonce un total de 14455 loges et 1508088 membres, alors que la somme des données détaillées pour chacune des organisations reconnues par le BIRM aux États-Unis est de 14455 loges et 1530958 membres, soit une différence de 22870 membres (BIRM, 1914, 392). 


\section{La production de francs-maçons et de loges}

\section{Graphique 1}

Les pôles régionaux de production maçonnique en 1914 (Francs-maçons recensés d'après BIRM, 1914)

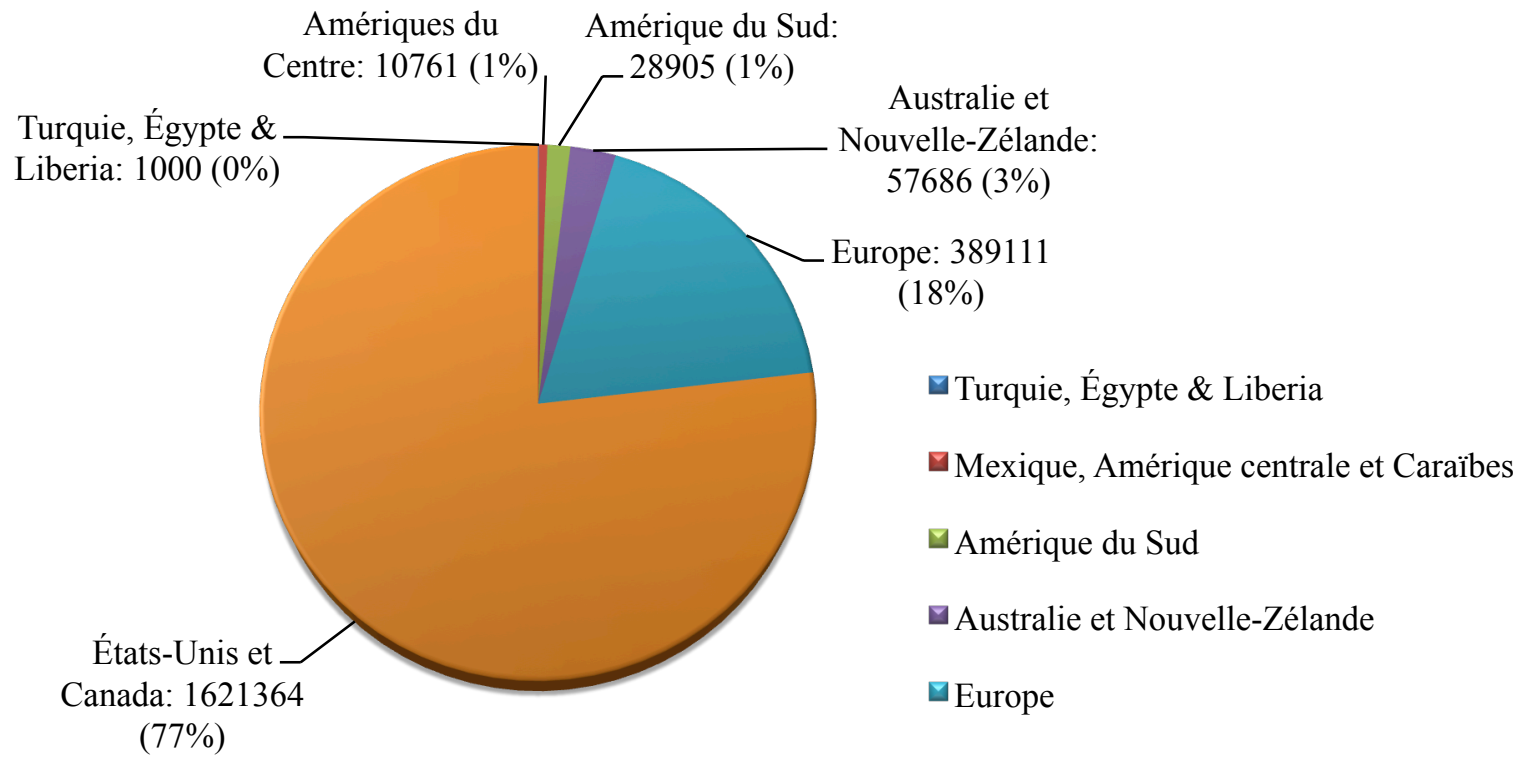

Etats-Unis et

Canada: 1621364

$(77 \%)$

Australie et Nouvelle-Zélande: $57686(3 \%)$

Europe: 389111

$(18 \%)$

$\square$ Turquie, Égypte \& Liberia

$\square$ Mexique, Amérique centrale et Caraïbes

$\square$ Amérique du Sud

$\square$ Australie et Nouvelle-Zélande

$\square$ Europe

Source : Bureau International de Relations Maçonniques, Annuaire de la Maçonnerie Universelle 8.

Graphique 2

Les pôles régionaux de production maçonnique en 1914

(Loges recensées d'après BIRM, 1914)

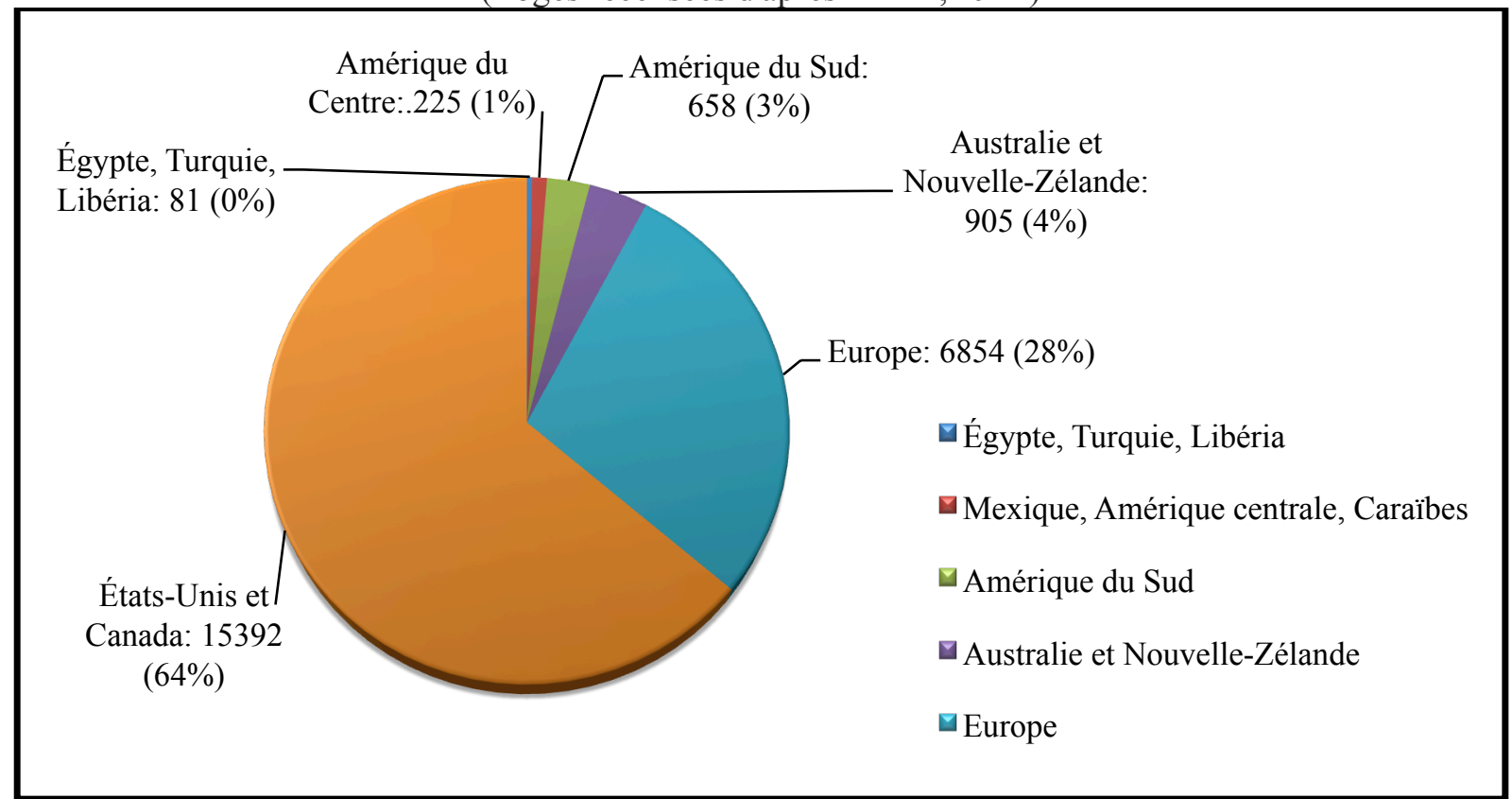

Source : Bureau International de Relations Maçonniques, Annuaire de la Maçonnerie Universelle 8. 
Les graphiques $\mathrm{n}^{0} 1$ et $\mathrm{n}^{\mathrm{o}} 2$ offrent un premier éclairage sur la production de francs-maçons et de loges dans le monde en 1914. Que montrent-ils? On distinguait au premier chef deux centres fondamentaux de poids inégaux: l'Amérique du Nord anglo-saxonne et l'Europe (occidentale pour l'essentiel). Les Grandes Loges recensées par le Bureau International aux États-Unis et au Canada ${ }^{26}$ regroupaient 1621364 membres répartis dans 15392 loges, soit environ 64\% des loges et $77 \%$ des francs-maçons recensés dans le monde. En Europe -occidentale pour l'essentiel- les Grandes Loges et Grands Orients recensés suivaient à la distance, avec 389111 membres (18\%) et 6854 loges (28\%).

Une seconde observation s'impose d'elle-même: l'Afrique et l'Asie ne jouaient qu'un rôle insignifiant dans la production maçonnique internationale. Le Bureau International de Relations Maçonniques ne détectait en Afrique que deux petites fédérations nationales: en Égypte (avec 500 membres) et au Liberia, où une grande loge fondée en 1867 réunissait à peine 250 membres. En Asie, seule était citée une fédération nationale, installé en Asie d'Europe: le Grand Orient Ottoman, avec ses 33 loges. Malgré une documentation imprécise à leur égard, on peut généreusement estimer leur poids démographique total à 81 loges et 1000 francs-maçons ${ }^{27}$.

Une troisième observation découle des précédentes: entre les centres et les périphéries de ce système-monde, l'Australie et l'Amérique latine occupaient des positions intermédiaires, minoritaires mais significatives. Les grandes loges recensées en Australie réunissaient 57686 membres (3\% du total mondial) et 905 loges (4\%). Les grandes loges et grands orients recensés en Amérique Latine regroupaient 2\% de la population maçonnique mondiale (39666 membres au total) et 4\% des loges (883 loges, dont 658 en Amérique du Sud et 225 dans les Amériques du Centre). Il faut toutefois noter que, dans le cas de l'Amérique latine, le recensement du Bureau était clairement sous-estimé, notamment pour le Mexique alors plongé dans une guerre révolutionnaire qui empêchait tout recensement exhaustif, il est vrai- mais aussi pour l'Argentine par exemple ${ }^{28}$. On peut penser que, en réalité, les sphères maçonniques australienne et latino-américaine étaient de volume comparable, sinon proche.

\footnotetext{
${ }^{26}$ Le Mexique est ici classé dans les "obédiences centraméricaines" en raison de son ambivalence: s'il appartient par sa position géographique à l'Amérique du Nord, il appartient à l'Amérique "latine" par sa position culturelle et maçonnique. Sa classification dans l'espace maçonnique centraméricain constitue donc une solution de compromis.

27 Source : BIRM, 1914. Sur la Turquie: Le BIRM indiquait pour le Grand Orient Ottoman 33 loges (dont 10 en instance) (BIRM, 1914, 369): on peut donc estimer sa population à un minimum entre 100 et 300 membres.

${ }^{28}$ Je reviendrai dans un prochain article sur l'Amérique latine, dont la situation a été analysée de manière approfondie dans Mollès, « Triangle atlantique », chap. 5.
} 


\section{La production de ramifications extraterritoriales}

\section{Graphique 3}

Les pôles régionaux de production maçonnique en 1914

(Origine nationale et situation territoriale des loges recensées in BIRM, 1914)

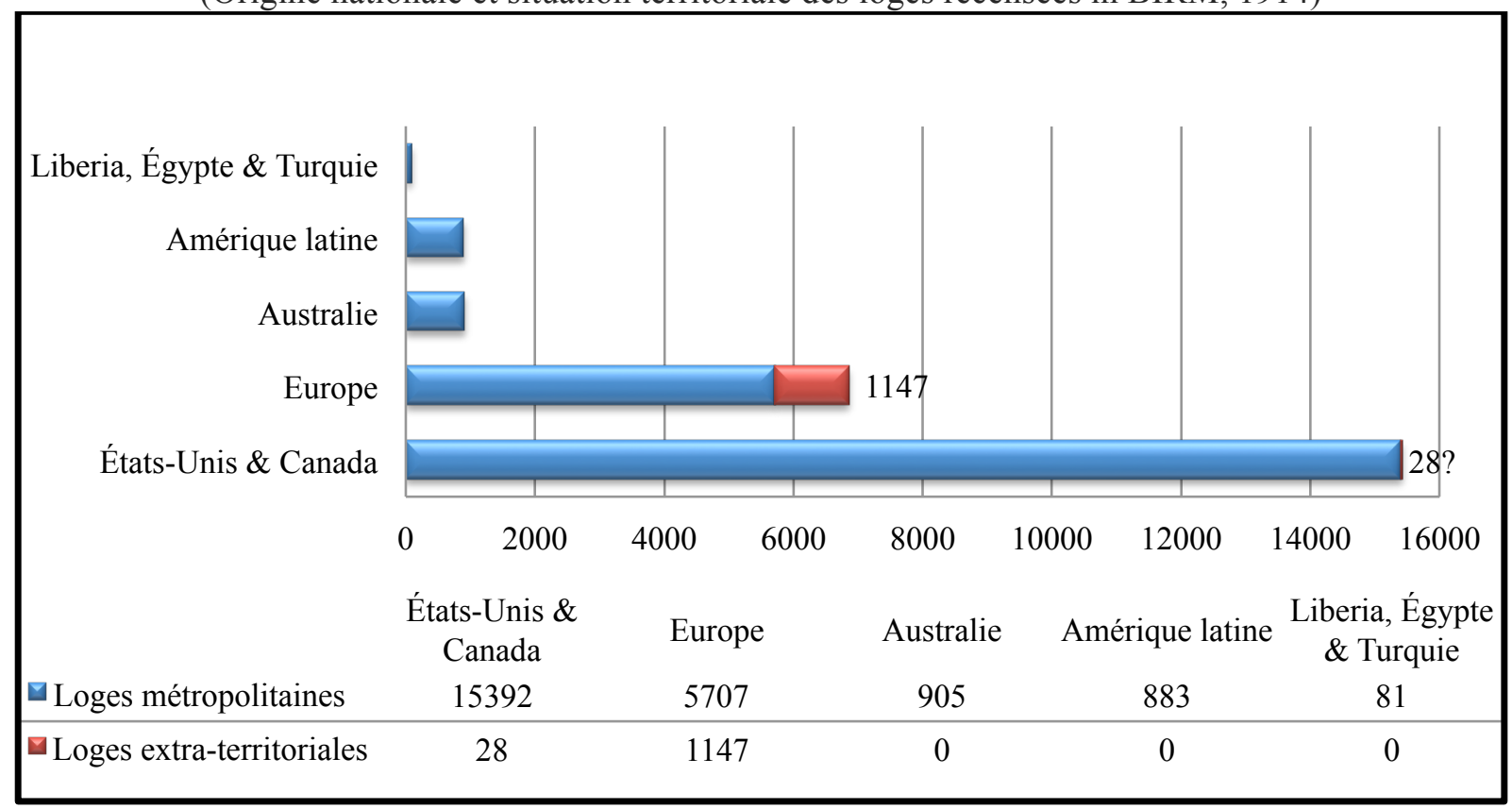

Source : Bureau International de Relations Maçonniques, Annuaire de la Maçonnerie Universelle 8.

Comme cela a été démontré ailleurs depuis des perspectives régionales ${ }^{29}$ ou globales $^{30}$, les ramifications extraterritoriales de l'Europe furent, dès les années 1720, le premier moteur de l'expansion maçonnique dans le monde. En 1914, l'Europe continuait-elle à produire ce type de loges extra-métropolitaines? Les obédiences européennes comptabilisées par le Bureau International patronnaient 5707 loges en métropole et 1147 loges extraterritoriales. La documentation ne permet pas, dans ce dernier cas, de recenser leurs effectifs humains.

Cette proportion était donc très significative. Comment s'organisait ce puissant dispositif? Apparemment, ce type de développement extraterritorial était le fait exclusif des Européens : c'est ce que laisse penser l'Annuaire de 1914. Mais, une nouvelle fois, peut-on lui accorder un crédit illimité ? On verra que d'autres sources permettent d'en douter, notamment dans le cas des États-Unis. Quoiqu'il en soit, la tendance structurelle semblait fermement établie : certaines franc-maçonneries européennes étaient disséminées dans le monde entier ; c'était là leur particularité presque exclusive.

\footnotetext{
${ }^{29}$ Sur les Caraïbes, voir le texte de Ferrer Benimeli, "Vías de penetración de la masonería en el Caribe", REHMLAC 1, no. 1 (May-Novembre 2009): 4-19 [cité 3 Octobre, 2014]: disponible dans http://rehmlac.com/recursos/vols/v1/n1/rehmlac.vol1.n1-jferrer.pdf

30 Par exemple dans Mollès, « Une Analyse Statistique (1717-1914) ».
} 


\section{Les équilibres géoculturels}

\section{La production de presse imprimée}

Graphique 4

La production de culture maçonnique dans le monde en 1914

(Origines régionales des 144 périodiques recensés d'après BIRM, 1914)

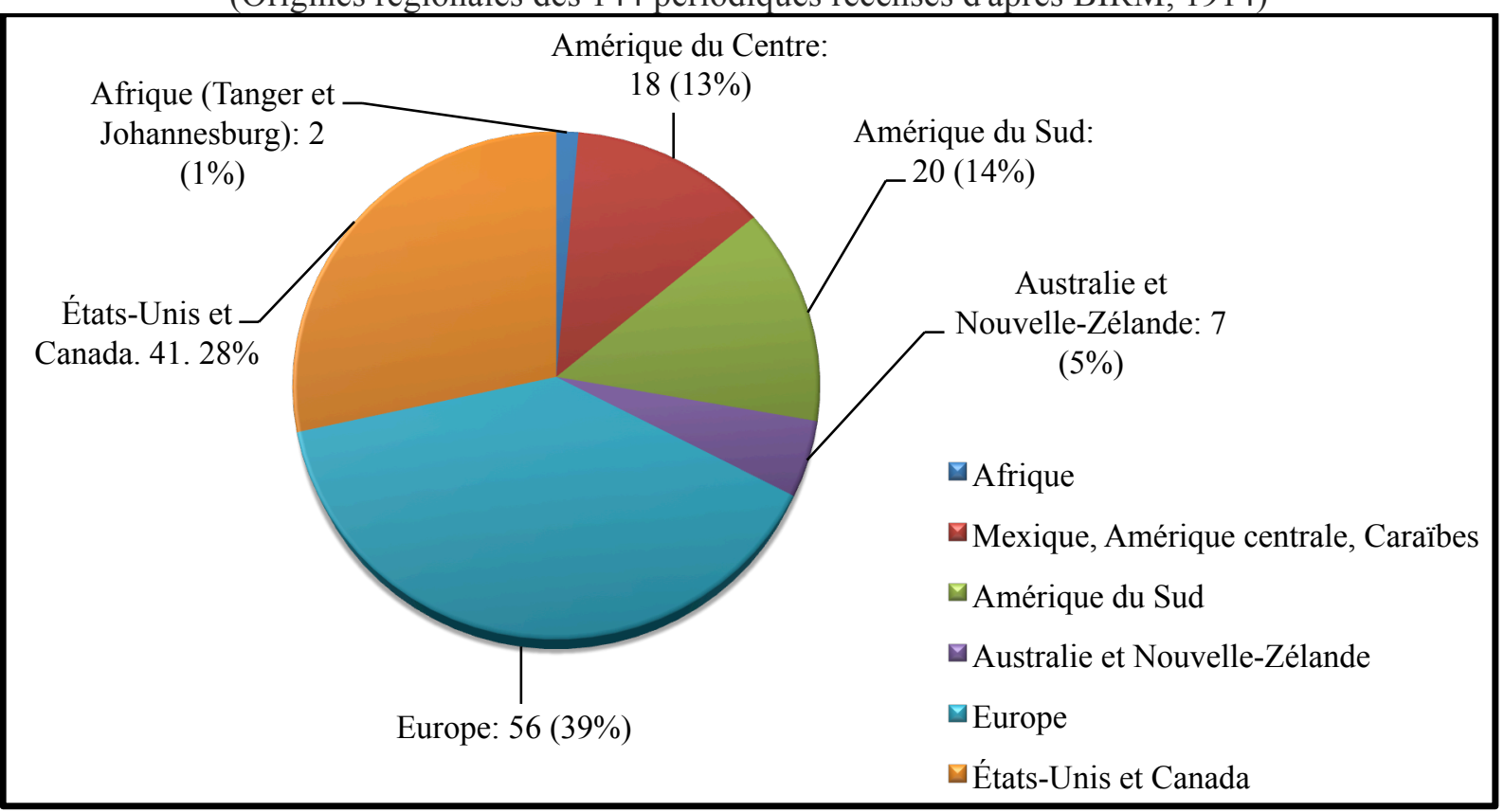

Source : Bureau International de Relations Maçonniques, Annuaire de la Maçonnerie Universelle 8.

Graphique 5

La production de culture maçonnique dans le monde en 1914

(Origines nationales des 144 périodiques recensés d'après BIRM, 1914)

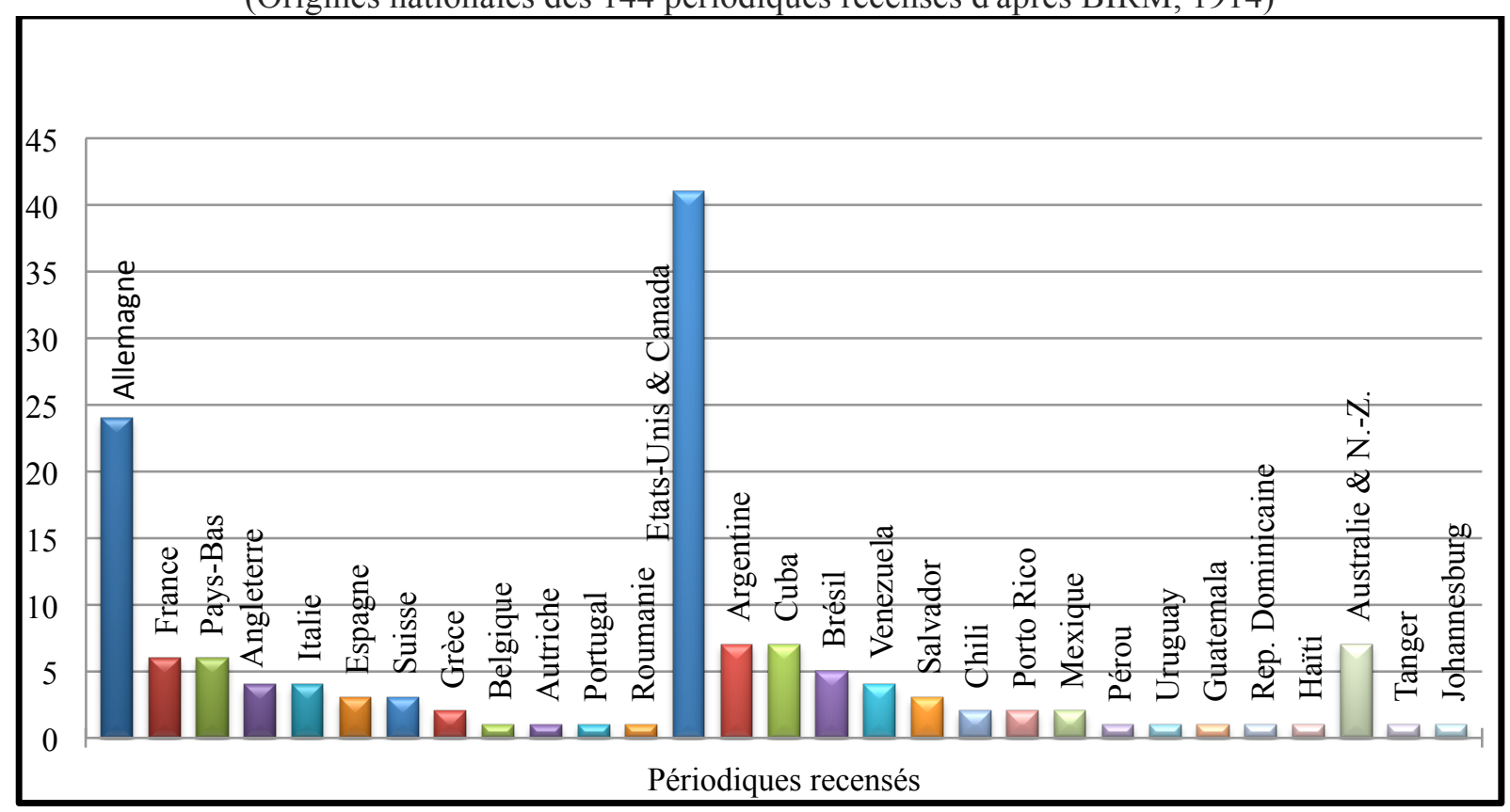

Source : Bureau International de Relations Maçonniques, Annuaire de la Maçonnerie Universelle 8. 
Une analyse de la presse maçonnique internationale confirme-t-elle cette économie générale ? L'Annuaire de la Maçonnerie Universelle ne prétendait pas recenser exhaustivement toutes les revues existantes à la veille de la Première Guerre mondiale. Il n'offrait qu'un échantillon, bien-sûr tributaire des réseaux de communication existants à l'époque d'abord, puis du réseau de correspondants postaux dont disposait le Bureau International de Relations Maçonniques. La tendance est indicative et sans nul doute existaient, hors de cette liste- de nombreuses publications de langues anglaise, française, allemande, espagnole ou portugaise.

Que montre cette liste ? Ces publications provenaient principalement d'Europe, d'Amérique du Nord, d'Amérique latine puis, dans une moindre mesure, d'Australie et de Nouvelle-Zélande. Ni la Turquie ni l'Asie n'apparaissaient. La tendance se confirme: l'Europe occidentale, l'Amérique anglo-saxonne et, l'Amérique latine et l'Australie étaient les épicentres de production et de diffusion de "la" culture maçonnique.

Un point mérite un bref commentaire. Comment interroger le dynamisme éditorial des Latino-Américains, qui tranchait avec la faiblesse relative des Nord-Américains en la matière. Avec seulement 2\% des francs-maçons recensés dans le monde, les Latino-Américains produisaient $27 \%$ des revues énumérées par le Bureau International. Treize nations en étaient la fabrique, emmenées par l'Argentine, Cuba, le Brésil et le Venezuela. Comparée à l'Australie (à peine $5 \%$ des périodiques recensés), l'Amérique latine semblait produire beaucoup plus. De ce constat naissent deux questions. Ce décalage n'alimente-t-il pas les doutes déjà soulignés sur la validité des chiffres du Bureau concernant l'Amérique latine ? En second lieu, cet enthousiasme éditorial était-il la manifestation d'un trait de mentalité particulier ? Traduisait-il par exemple une propension à la prise de parole collective au sein de la sphère publique beaucoup plus forte que chez les Anglo-Saxons? 


\section{Franc-maçonneries latines et anglo-saxonnes}

\section{Graphique 6}

Latins et Anglo-Saxons dans le monde maçonnique en 1914

Une estimation corrigée d'après BIRM, 1914

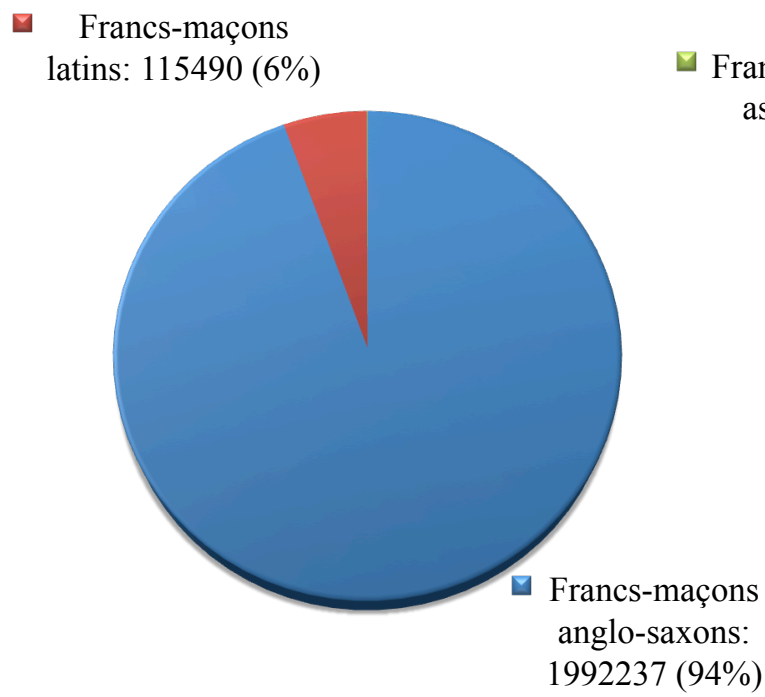

Source : Bureau International de Relations Maçonniques, Annuaire de la Maçonnerie Universelle 8.

Graphique 7

Latins et Anglo-Saxons dans le monde maçonnique en 1914

Une estimation corrigée d'après BIRM, 1914

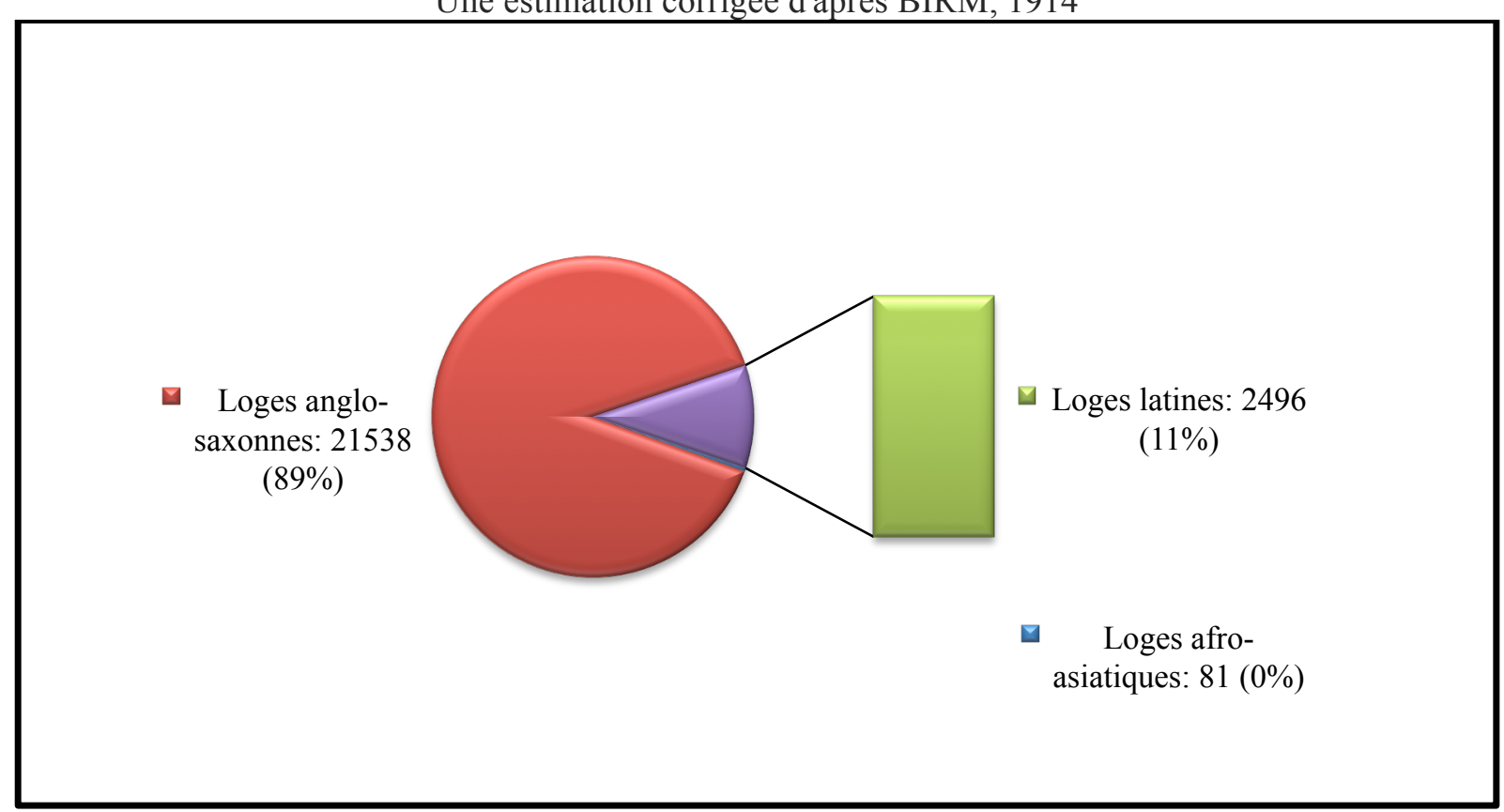

Source : Bureau International de Relations Maçonniques, Annuaire de la Maçonnerie Universelle 8. 
Selon les sources unanimes, deux groupes géoculturels coexistaient au sein de la sphère maçonnique internationale à cette époque: "Latins" et "Anglo-Saxons". Cette distinction fut validée par le Bureau International, dont l'Annuaire distinguait explicitement (et au premier plan) entre Latins, Britanniques et Allemands (ce troisième groupe comprenant les Scandinaves) $)^{31}$. Sans entrer dans une analyse comparée de leurs qualités, notons que, d'après les données utilisées, environ $89 \%$ des loges recensées s'inscrivaient dans l'un des courants anglo-saxons, tandis qu'environ $11 \%$ dérivaient d'une tradition latine. Ce déséquilibre s'accentuait au regard du nombre d'adeptes recensés : 94\% de francs-maçons anglo-saxons contre $6 \%$ de francs-maçons latins. Ces chiffres posent question sur les pratiques particulières de ces deux groupes: de toute évidence, les Anglo-Saxons préféraient les loges nombreuses; en revanche, les Latins optaient pour des loges plus intimes. La parole pouvait-elle circuler dans les mêmes conditions dans une loge de 50 membres et dans une loge de 500 membres ? D'où une question de fond: loges latines et loges anglo-saxonnes possédaient-elles la même fonction et la même vocation en matière de circulation et de prise de parole?

Structure linguistique du monde maçonnique d'après (BIRM, 1914)

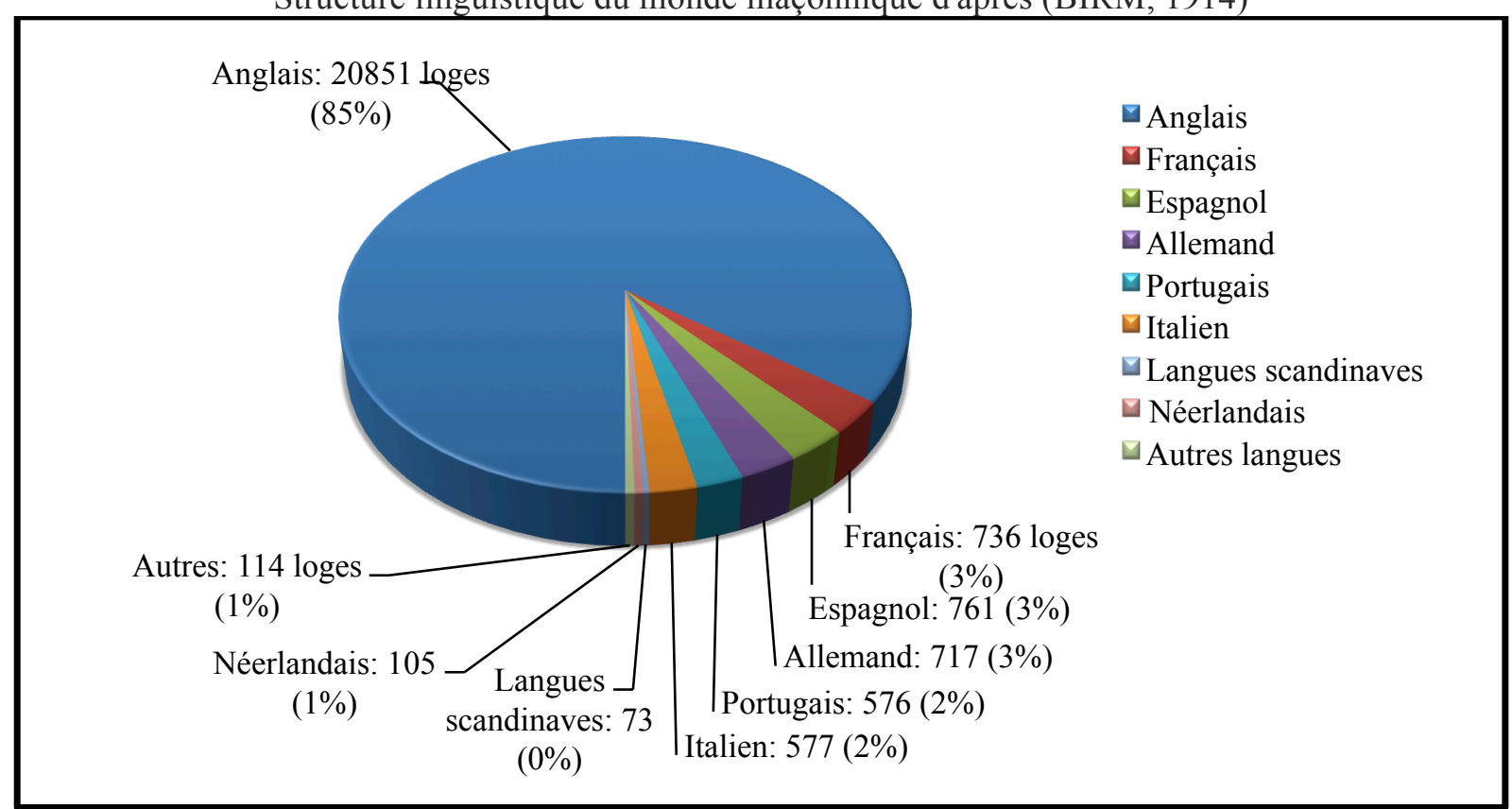

Source : Bureau International de Relations Maçonniques, Annuaire de la Maçonnerie Universelle 8.

Les données linguistiques confirment globalement ces équilibres. Environ $86 \%$ des loges recensées par le Bureau International travaillaient en langue anglaise. Les loges de langues allemande, scandinaves et néerlandaise pesaient ensemble un peu plus de $4 \%$. Les langues latines étaient pratiquées par environ $10 \%$ des loges recensées, à travers le français

\footnotetext{
31 Voir le tableau général dans BIRM, 1914, pp. 391-393.
} 
(3\%), l'espagnol (2\%), le portugais (2\%), l'italien (3\%). Les langues roumaine, arabe, grecque, turque et serbo-croate ne représentaient qu'un pourcentage résiduel.

On peut émettre quelques réserves. Ainsi, la somme des loges de langue espagnole recensées par le Bureau pour 1914 est de 493, Amérique latine comprise. En réalité, des éléments objectifs attestent de la sous-valorisation du groupe ibérique. Dans une section marginale, l'Annuaire citait quelques obédiences «non régularisées »: la plupart étaient de langue espagnole (Mexique, Cuba, Argentine, etc.) ${ }^{32}$. Aucune information statistique n'était fournie à leur sujet. En conséquence, j'ai procédé à une estimation minimale de leurs effectifs. Cette estimation aboutit à un total de 761 loges de langue espagnole. On pourrait procéder de même pour les loges de langue portugaise, comme on le verra au regard du cas brésilien.

\section{Conclusion}

À la veille de la Première Guerre mondiale, la sphère maçonnique mondiale réunissait plus de deux millions d'adeptes et vingt-quatre mille loges, selon le Bureau International de Relations Maçonniques dont les Annuaires -bien que méconnus- constituent la source probablement la plus complète disponible pour une analyse globale. Certes, on l'a vu, des logiques diplomatiques conduisirent les auteurs de ces Annuaires à en exclure les loges afro-américaines, mixtes, féminines ou encore certaines loges masculines (notamment en Amérique latine). Celles-ci ne pouvaient toutefois sensiblement modifier l'économie générale du monde maçonnique, couvert à au moins 95\% par cette radiographie. La franc-maçonnerie formait donc une sorte de société civile internationale. Malgré son volume considérable, cet iceberg est globalement demeuré dans l'ombre. Comment se cristallisait-il ?

La sphère maçonnique était paneuropéenne. Apparue en Europe occidentale au début du XVIII ${ }^{\mathrm{e}}$ siècle, elle s'était élargie dans le sillage de l'expansion des puissances et des sociétés européennes. À la fin du XIX ${ }^{\mathrm{e}}$ siècle, ses seules véritables usines de production étaient localisées dans les grandes aires de colonisation et de peuplement paneuropéen: l'Europe occidentale, les Amériques (du Nord, du Centre, du Sud) et l'Australie.

Cet univers était traversé par une tension entre national et international. D'un côté, ces 24000 loges fournissaient un extraordinaire réseau de sociabilité et de circulation internationale. De l'autre côté, elles émanaient de pôles de production nationaux. Ces pôles étaient des fédérations (plus d'une centaine de grandes loges et de grands orients) dont la souveraineté s'exerçaient sur un territoire national déterminé, concrètement sur le territoire d'un des quarante États-nations mentionnées (ou, dans le cas de l'Australie et du Canada, d'une communauté politique partiellement autonome intégrée au Commonwealth). Ainsi, d'une certaine manière, les réseaux maçonniques fournissaient-ils une articulation communes aux sociétés paneuropéennes, permettant les échanges, les transferts, les harmonisations, les

\footnotetext{
${ }^{32}$ Ces grandes loges "non régularisées" et non comptabilisées étaient: "Nacional de Cuba", "del Estado de Coahuila", "del Estado de Chihuahua, "del Estado de Nuevo León", "del Estado de Oaxaca", "del Estado de Puebla", "del Estado de Tamaulipas", "Unida Mexicana del Estado de Veracruz", "Valle de México", "Lealtad de San Salvador) : BIRM, 1914.
} 
adaptations culturelles. Ils conféraient ainsi puissance et souplesse à la civilisation paneuropéenne dont l'expansion globale touchait son apogée.

En dehors de l'Europe occidentale, des Amériques et de l'Australie, on distinguait deux types de situations: le monde colonial et les grands empires orientaux. Le monde colonial -où les puissances européennes s'étaient partagées d'immenses territoires- ne s'intégrait à la sphère maçonnique que par l'intermédiaire des quelques 1200 loges européennes établies pour l'essentiel en Orient, dans le Pacifique et en Afrique (loges extraterritoriales). On pourrait certes souligner les cas de la Turquie, de l'Égypte et du Liberia où trois petites franc-maçonneries autonomes doivent-être signalées pour une future approche. Ces dispositifs servirent-ils d'articulation entre les élites locales et les élites paneuropéennes ?

Par ailleurs, les grands empires orientaux -dynamiques comme le Japon ou décadents comme la Russie et la Chine- semblaient hostiles à la franc-maçonnerie qui y semblait absente, (à l'exception de quelques loges européennes extraterritoriales très particulières en Chine et au Japon). Cela reflétait-il une hostilité aux influences de la modernité libérale occidentale? Cela reflétait-il aussi l'anomie des sociétés orientales qui -soumises à des régimes de contrôle social autoritaires, aristocratiques et répressifs- ne pouvaient créer un espace public moderne, préalable nécessaire à tous les types de sociabilité démocratique et, notamment, maçonnique?

Les quatre pôles régionaux de production maçonnique étaient de dimensions inégales. Quels étaient les centres et les périphéries de ce système-monde ? L'Amérique du Nord et l'Europe occidentale produisaient entre $92 \%$ et $95 \%$ des loges et des francs-maçons recensés, dont respectivement $64 \%$ et $77 \%$ pour les États-Unis et le Canada, et respectivement $28 \%$ et $18 \%$ pour l'Europe. Compensant cet écart important, l'Europe produisait cependant -en plus de ses loges métropolitaines- des loges extraterritoriales. Ajouté à son importance symbolique de creuset originel de la franc-maçonnerie, ne s'agissait-il pas d'un facteur de puissance et de rayonnement, un dispositif international de soft power? Dans ce système-monde, l'Australie et l'Amérique Latine s'inscrivaient entre le centre et la périphérie: d'un côté elles produisaient des quantités non négligeables de loges et de francs-maçons. De l'autre, elles abritaient une quantité significative de loges européennes extraterritoriales.

Sur le plan géoculturel, les estimations réalisées mettent en évidence que les traditions « anglo-saxonnes » représentaient environ $89 \%$ de l'effectif maçonnique mondial, tandis que les traditions «latines » représentaient une forte minorité d'environ 11\%. Cette tendance, estimée à partir des statistiques concernant les loges et les membres, semble confirmée par l'analyse de la structure linguistique du monde maçonnique, cette Babel paneuropéenne. Quel fut l'impact de la Première Guerre mondiale sur ces équilibres, de l'Europe occidentale aux Amériques en passant par l'Afrique, l'Asie et le Pacifique?

Bien-sûr, il s'agit d'un idéal-type, d'une simplification de la réalité vivante et donc fluctuante. Par exemple, selon l'utilisation que le Bureau International de Relations Maçonnique faisait explicitement de cette typologie, le Grossorient des Königreichs der Niederlanden (Pays-Bas) et la Grande Loge Suisse Alpina s'inscrivaient dans la tradition latino-européenne. Or, une observation plus fine ne montrerait-elle pas que ces maçonneries protestantes de tradition libérale se trouvaient en quelque sorte à la charnière des deux 
groupes géoculturels ? D'où une question légitime : ce cas de figure, cette mixité variable se répétait-elle ailleurs, par exemple en Amérique Latine?

$\mathrm{Au}$ total, après avoir défini le volume de la sphère maçonnique internationale et ses équilibres régionaux, on distingue quatre groupes de situation: peut-on parler à leur sujet de centres (Amérique anglo-saxonne et Europe occidentale), de semi-périphéries (Amérique Latine et Australie), de périphéries (Afrique, Asie) et de zones fermées à la navigation, sauf cas particulier (les empires orientaux). Chacune de ces configurations est justiciable, notamment au regard de la Première Guerre mondiale, d'une analyse particulière; celle-ci sera d'autant plus utile qu'elle sera capable d'articuler différentes échelles d'analyse, du local au global.

\section{Bibliographie}

Bureau International de Relations Maçonniques. Annuaire de la Maçonnerie Universelle 8. Berne, Suisse: Imp. Büchel, 1914.

Coen, Jorge. "Recuerdos de Mi Iniciación". En : Verbum, Revista Masónica Argentina Fundada En 1934 1, no. 6 (Novembre 15, 1934): 4.

Ferrer Benimeli, José Antonio. "Vías de penetración de la masonería en el Caribe". Dans : REHMLAC 1, no. 1 (May-Novembre 2009) : 4-19. Disponible dans http://rehmlac.com/recursos/vols/v1/n1/rehmlac.vol1.n1-jferrer.pdf

Ferrer Benimeli, José Antonio. "Aproximación a la Historiografía de la Masonería Latinoamericana". Dans: REHMLAC 4, no. 1 (May-Novembre 2012) : 2-120. http://www.rehmlac.com/recursos/vols/v4/n1/rehmlac.vol4.n1-jferrer.pdf

Ferrer Benimeli, José Antonio. "Reseña de La Franc-Maçonnerie dans les ports, editado por Cécile Révauger y Éric Saunier”. Dans : REHMLAC 5, no. 1 (May-Novembre 2013): 228-236. Disponible dans http://rehmlac.com/recursos/vols/v5/n1/rehmlac.vol5.n1-jferrer.pdf

Gould, Robert Freke. Military Lodges: The Apron and the Sword or Freemasons under Arms. Accounts of Lodges in Regiments and Ships of War and Famous Soldiers and Sailors of All Countries Who Have Belonged to the Society, Together with Biographies of Distinguished Military and Naval Brethren, and Anecdotes Showing the Influence of Masonry in Warfare. By Robert Freke Gould, Past Senior Gran Deacon of England, P.M. n 92 and 2076 (London), 153 (Gibraltar), 570 (Shanghai) and 743 (1st Batt., East Surrey Regiment, Etc. Londres: Gale \& Polden Ltd., 1899.

Le Bureau international de relations maç. ., Son histoire (1889-1905). Rapport présenté à la Grande Loge Suisse Alpina le 25 juin 1905. Berne, Suisse: Bureau International des Relations Maçonniques - Imprimerie Büchler \& Co., 1905.

Ligou, Daniel, ed. Dictionnaire de la franc-maçonnerie. 4th ed. Paris: Presses Universitaires de France, 1998.

Martínez Esquivel, Ricardo. "Hacia la construcción de una historia social de la masonería en Centroamérica". Dans: Estudios 27 (2013). Disponible dans http://revistas.ucr.ac.cr/index.php/estudios/article/view/12703 
Mollès, Dévrig. « Triangle atlantique et triangle latin: l'Amérique latine et le système-monde maçonnique (1717-1921) (Éléments pour une histoire des opinions publiques internationales). » Thèse de doctorat en histoire contemporaine, Université de Strasbourg, 2012.

Mollès, Dévrig. «L'histoire Globale et La Question Maçonnique: Éléments Pour Une Analyse. » Dans : REHMLAC 6, no. 1 (May-Novembre 2014): 3-33. Disponible dans http://rehmlac.com/recursos/vols/v6/n1/rehmlac.vol6.n1-dmolles.pdf

Mollès, Dévrig. « Le Triangle Atlantique: Émergence et Expansion de La Sphère Maçonnique Internationale. Une Analyse Statistique (1717-1914)». Dans : Nuevo Mundo Mundos Nuevos. Nouveaux Mondes Mondes Nouveaux - Novo Mundo Mundos Novos - New World New Worlds 14 (2014). Disponible dans http://nuevomundo.revues.org/67498

Perrelet, Bernard. Le Bureau International de Relations Maçonniques: Organisation, but, activité. Berne - Neuchâtel: Bureau International des Relations Maçonniques Imprimerie Büchler \& Co., 1913.

Révauger, Cécile. «Les Femmes et La Franc-Maçonnerie, Des Origines À Nos Jours.» Dans : REHMLAC 4, no. 2 (Décembre 2012-Avril 2013 2012): 11-31. Disponible dans http://rehmlac.com/recursos/vols/v4/n2/rehmlac.vol4.n2-crevauger.pdf

Révauger, Cécile. Noirs et francs-maçons aux États-Unis. 2nd ed. édition revue et augmentée. Paris: Dervy, 2014.

Rouquié, Alain. L'Amérique latine: Introduction à l'Extrême-Occident. Paris: Seuil, 1987. 